\title{
La Web Semántica y los archivos de televisión: estado de la cuestión
}

\author{
Rodrigo Sánchez Jiménez \\ Jorge Caldera Serrano \\ Iuliana Botezan
}

Recibido: 28 de enero de 2016

Aceptado: 1 de marzo de 2016

DOI: http://dx.doi.org/10.5209/rev_CDMU.2016.v27.n1.51979

\section{Resumen}

Se analizan y describen las principales líneas de trabajo de la Web Semántica en el ámbito de los archivos de televisión. Para ello, se analiza y contextualiza la web semántica desde una perspectiva general para posteriormente analizar las principales iniciativas que trabajan con lo audiovisual: Proyecto MuNCH, Proyecto S5T, Semantic Television y VideoActive.

\section{Palabras clave}

Web Semántica / Archivos de televisión / Información audiovisual / Multimedia / Ciencias de la Documentación

\section{The Semantic Web and television archives: state of affairsy}

\section{Abstract}

This paper reviews the main lines of work around the use of Semantic Web technologies in the field of television archives. To that end, we sumarize general aspects of the Semantic Web and then describe and analyze the most prominent initiatives that deal with audiovisual information using such technologies: MuNCH Project, S5T Project, Semantic Television and VideoActive.

\section{Keywords}

Semantic Web / Television Archives / AV information / Multimedia / Information Science 


\section{Introducción. La Web Semántica desde la perspectiva de la Documentación}

Tim Berners-Lee define la Web Semántica como "...una extensión de la Web actual en la que se proporciona a la información un significado bien definido, lo que permite a la gente y las computadoras trabajar en cooperación" (Berners-Lee, 2004).

Para proporcionar a la información un significado bien definido es necesario representarla o describirla de una forma explícita. En el ámbito de la Web Semántica se utiliza un conjunto de reglas reunidas en el modelo RDF, o Resource Description Framework (W3C, 2011b) para llevar esto a cabo. RDF no está constituido por un conjunto delimitado de campos que se utilizan en la descripción de recursos, sino que proporciona la lógica más básica, la estructura y el paradigma con el que especificar la manera en que se hace la descripción. No se trata por tanto de un estándar equivalente a MARC-XML, sino de un modelo más genérico y abstracto, equivalente por ejemplo al modelo entidad-relación que se utiliza para crear la estructura en tablas, columnas y registros en la mayoría de las bases de datos.

RDF es un lenguaje que nació para fundamentar la descripción de recursos mediante metadatos en el ámbito de Internet (Manola y Miller, 2004). Por su parte, los metadatos no son otra cosa que el resultado de la adaptación y evolución de los principios organizativos y descriptivos de la Biblioteca y el Centro de Documentación a Internet. En nuestra opinión, en tanto que RDF se refiera a la descripción de documentos, debería ser considerado como una herramienta más para los documentalistas y los investigadores en el ámbito de las Ciencias de la Documentación (Blanco y Póveda, 2015).

De hecho el área de la Biblioteconomía y Documentación hace mucho que traspasó el área de la descripción y representación de documentos y se adentró en la de la descripción y representación de personas e instituciones en su función de autores de documentos. Un ejemplo evidente de esto son los ficheros de autoridades. En los ficheros de autoridades no sólo se normaliza una entrada, sino que se proporcionan datos sobre las fechas de nacimiento y defunción, la lengua y la nacionalidad del autor 
(IFLA-UBCIM, 2001). Se representa por tanto a los autores en sí mismos, con sus propias características.

En el ámbito de las Ciencias de la Documentación, existen por tanto ejemplos y precedentes de descripción de recursos (y no sólo de documentos) por lo que la diferencia esencial entre las prácticas descriptivas de la Web Semántica y las que se han consolidado en Biblioteconomía y Documentación no reside en esta diferencia entre recurso y documento, sino que reside en la formalización de las descripciones para que sean entendidas por máquinas, y no sólo procesadas, como ocurre por ejemplo con MARC-XML.

En palabras de Berners-Lee (2001): "For the semantic web to function, computers must have access to structured collections of information and sets of inference rules that they can use to conduct automated reasoning". Sobre esta base, en Biblioteconomía y Documentación ya se ha trabajado con intensidad sobre la creación de colecciones estructuradas de información y lo que falta por tanto son las reglas de inferencia.

El papel del Consorcio W3C en lo que se ha dado en llamar Web Semántica es fundamental, dado que la idea original parte del líder del Consorcio (Tim Berners-Lee) y básicamente todos los estándares importantes han sido creados o sancionados en el seno de dicha organización. La Web Semántica se apoya con fuerza en el paradigma de Representación del Conocimiento de la Inteligencia Artificial, así como en el razonamiento sobre dicha representación. En palabras de Berners-Lee (2001):

\footnotetext{
"The challenge of the Semantic Web, therefore, is to provide a language that expresses both data and rules for reasoning about the data and that allows rules from any existing knowledge-representation system to be exported onto the Web."
}

El desarrollo actual de los estándares e iniciativas relativos a la Web Semántica, así como lo sustancial de la literatura científico-técnica sobre la materia son producto del trabajo de investigación y desarrollo de diversas disciplinas, entre las que se encuentra la 
Documentación, pero en las que dominan las Ciencias de la Computación. La Documentación, desde la perspectiva de las Ciencias Sociales, y partiendo de un corpus científico fundado en la investigación en Información, debería tener un papel mucho más destacado en el desarrollo de las teorías, técnicas y herramientas para la representación y gestión del Conocimiento.

Desde nuestro punto de vista, los centros de documentación deberían constituir un banco de pruebas fundamental para la experimentación de los presupuestos de la Web Semántica. Muchas de las utilidades que se supone conlleva la adopción del paradigma de Berners-Lee pueden ser validadas en un entorno de uso intensivo de la información como es un centro de documentación.

\section{La Web Semántica aplicado a la información audiovisual televisiva}

En general, la investigación y la difusión de resultados en lo referente al tratamiento de documentación audiovisual se hayan condicionadas por el hecho de que la documentación fuente y las herramientas documentales son propiedad de alguna de las televisiones, fundamentalmente privadas (Caldera, 2015). Esto reduce el campo de acción (salvo en casos particulares) al ámbito de las televisiones públicas o de los organismos públicos encargados de la preservación (Blanco, 2015). Si a esto le añadimos que la aplicación de técnicas derivadas de la Web Semántica al campo del audiovisual (desde una perspectiva documental, al menos) es escasa (Caldera y Sánchez, 2008), tenemos que el panorama actual se vertebra entorno a un número limitado de proyectos dotados de bastantes medios y en los que colaboran instituciones fundamentalmente públicas relacionadas con los archivos audiovisuales televisivos y universidades o institutos de investigación.

Se han revisado aspectos más genéricos relativos a la aplicación de tecnologías de la Web semántica sobre documentación Multimedia con el objetivo de ampliar la base bibliográfica e introducir algunas líneas de investigación potencialmente aplicables al problema de los archivos televisivos. Lo que sigue es una relación de las principales líneas de investigación sobre aplicación de tecnologías de la Web Semántica a la 
documentación multimedia y los archivos televisivos tras lo que se introducen las aportaciones previas del candidato.

\section{Multimedia y Web Semántica}

Existe una abundantísima literatura que se refiere a la aplicación de tecnologías de la Web Semántica (ontologías, razonamiento automático, metadatos, RDF...etc.) рara problemas relativos a la gestión y recuperación de documentación audiovisual y multimedia. No es posible resumir adecuadamente las más de 300 referencias que hemos extraído de la "Web of Science" sobre estos aspectos, aunque intentaremos dar cuenta a continuación de las tendencias más destacadas, así como de las obras más representativas de las mismas.

\section{a) Web Semántica Multimedia}

La proliferación de contenidos audiovisuales y de imagen en Internet en los últimos tiempos, junto con la progresiva materialización de tecnologías y conceptos de la Web Semántica ha hecho surgir un concepto innovador en la investigación en documentación audiovisual en el ámbito de la Web, la "Web Semántica Multimedia”. El concepto fue utilizado por primera vez por Grosky, Sreenath y Fotouhi en 2002 (Grosky et al, 2002) y se basa en la interrelación de recursos multimedia de acuerdo con los principios de la Web Semántica.

Esta perspectiva se ha aplicado fundamentalmente a la documentación sonora (Tummarello, 2005 y Hogan, 2005) y musical (Moutselakis, 2009), aunque Polo (2005), sobre el proyecto “M-ADVANTAGE", y Khriyenko (2007) con "4l”, han extendido este concepto hacia la creación de plataformas para el desarrollo de las ideas de la Web Semántica aplicadas a la navegación e interrelación de recursos multimedia, sin éxito hasta la fecha, no obstante.

b) Multimedia semántico 
Asociado a este concepto encontramos el de "Semantic Multimedia", término que predomina hoy en la literatura científica y técnica para referirse a la aplicación de los principios de la Web semántica a la descripción, tratamiento y recuperación de documentación multimedia. Como el propio nombre sugiere, se trata de introducir la semántica dentro del propio documento multimedia, poniéndose por tanto el énfasis en la descripción de recursos.

El problema fundamental de la descripción de contenidos multimedia es probablemente el de salvar la "brecha semántica" (Briassouli et al, 2007) que media entre las descripciones de bajo nivel generadas automáticamente por parte de sistemas de reconocimiento de imagen y los conceptos manejados por el usuario y el documentalista (más complejos, de alto nivel) para recuperar documentación multimedia.

Se trata por tanto de superar una característica inherente de la documentación audiovisual (y por extensión multimedia) que es la de la opacidad de la misma (Hidalgo Goyanes, 1999). La investigación en esta línea puede a medio plazo proporcionar importantes beneficios en la semi-automatización de la descripción.

Debido a la complejidad de la tarea, se trata de un área de investigación y desarrollo tecnológico que implica grandes dosis de ingeniería, y de multidisciplinaridad, por lo que agrupa a científicos y técnicos del área del procesamiento de señales, reconocimiento de patrones, análisis automatizado de la imagen y recuperación de información que están haciendo un uso cada vez más extensivo de las tecnologías propias de la Web Semántica (Avrithis et. al, 2008). Este es en realidad un tema de investigación no bien resuelto (por su complejidad) y por lo tanto todavía abierto. El ejemplo más cercano a nuestra perspectiva (la de los archivos televisivos) nos lo proporcionan Hollink et al (2009).

\section{c) Sistemas de recomendación}

Alam, Iqbal, Noll y Chowdhury (2009) se centran en la difusión de información audiovisual a través de descodificadores de TDT (o cable). La mayor parte de los artículos 
que se presentan sobre este tipo de aparatos se refieren a la personalización de la programación y al establecimiento de perfiles de usuario para desarrollar sistemas de recomendación de contenidos. Desarrollan una ontología de perfiles de usuarios y otra para importar los contenidos de TV-Anytime (metadatos sobre la programación que no se utilizan en España, donde utilizamos XMLTV).

Bellekens, Houben, Aroyo, Schaap, y Kaptein (2009) trabajan también sobre ontologías y TDT, pero se centran en el concepto de guía personalizada de televisión. La ontología sirve fundamentalmente para crear los modelos de usuario, mientras que los contenidos se extraen automáticamente del EPG. Este es un enfoque similar al de Brut et al (2008), aunque en este caso también se utilizan técnicas de recuperación de información como el algoritmo de k-vecinos.

Blanco-Fernández et al (2008) utilizan de nuevo una ontología OWL y el estándar TVAnytime para la descripción de contenidos y el establecimiento de procedimientos de razonamiento automático para crear el sistema de recomendación AVATAR (BlancoFernández et al, 2004), que enfatiza la personalización de contenidos televisivos.

d) MPEG-7 y Ontologías

MPEG-7 es un estándar ISO desarrollado por el Moving Picture Experts Group (que también desarrolló estándares fundamentales hoy como MPEG-1 o MPEG-2). Es una especificación con vocación multimedia, aunque dada la complejidad de los medios audiovisuales, una parte importante de dicho estándar se refiere a la descripción de imágenes en movimiento (Polo, 2011).

El nombre formal de la especificación es "Multimedia Content Description Interface" (Martínez, 2004). MPEG-7 permite la descripción de documentos multimedia desde múltiples perspectivas, que podemos resumir en características de bajo nivel, características estructurales, formales y de contenido. La parte del estándar que se refiere a los esquemas de descripción multimedia tiene 800 páginas (ISO, 2001), lo que da una idea de su envergadura. La unidad básica para la descripción es el "esquema de 
descripción" (DS), término que en realidad no se refiere a un esquema XML completo, sino a un fragmento de la estructura del mismo. Un esquema de descripción y que se corresponde (grosso modo) con una clase de entidad, aunque ésta no se formaliza tal y como se haría en una ontología.

Desde el punto de vista del contenido, quizá la perspectiva más interesante para el tratamiento documental, MPEG-7 proporciona esquemas de descripción para objetos, personas, lugares, individuos, grupos y momentos. Cada uno de ellos tiene sus correspondientes atributos, para los que en ocasiones existen listas de valores controlados.

Desde el punto de vista de la descripción formal se pueden utilizar esquemas de descripción para títulos, autores, formas, formatos, géneros...etc. Así mismo se ofrecen esquemas de descripción muy detallados para dar cuenta de la estructura temporal o espacial de la imagen, lo que permite asociar entidades a las coordenadas marcadas por la región de la imagen y la línea de tiempo.

Según Dasiopoulou, Tzouvaras, Kompatsiaris y Strintzis (2010) el dominio de la documentación audiovisual está bien cubierto por MPEG-7 pero el esquema XML del estándar carece del formalismo necesario. Esto habría ocasionado el surgimiento de varias iniciativas (ninguna definitiva) para intentar hacer compatibles las descripciones realizadas sobre la base de MPEG-7 con los principios de la Web Semántica. El trabajo de Dasiopoulou, Tzouvaras, Kompatsiaris y Strintzis (2010) ofrece una revisión exhaustiva de la literatura sobre el tema y resume con precisión los problemas fundamentales relativos a esta línea de investigación. Este trabajo nos ha servido de guía para analizar las iniciativas más importantes sobre la materia, aunque en nuestro caso hemos profundizado tan sólo en los aspectos referentes a la gestión y descripción de contenidos, dejando de lado los aspectos estructurales y de bajo nivel de la especificación.

Las diferentes propuestas para introducir MPEG-7 se basan en el principio común de representar total o parcialmente los esquemas de descripción de MPEG-7 mediante una 
o varias ontologías que se refieren a las características de bajo nivel del audio y el vídeo, la estructura y la descripción de contenidos, pero en el marco de arquitecturas que permiten la introducción de extensiones de estos aspectos básicos desde la perspectiva de un dominio o una aplicación concreta.

El proyecto Harmony utiliza un esquema RDF de carácter genérico para cubrir los aspectos fundamentales de MPEG-7 (Hunter, 2001), lo que incluye las descripciones de bajo nivel del video y el audio, así como la estructura. Sin embargo, la descripción del contenido se lleva a cabo a través de la ontología ABC (Lagoze y Hunter, 2001). ABC proporciona un conjunto de clases abstractas y propiedades que forman el modelo de compatibilidad, mientras que se espera que para cada dominio específico se desarrolle una ontología que extienda las clases originales refinando su significado y aplicándolo.

Se trata por tanto de una "meta-ontología" que proporciona los conceptos básicos y una definición formal extensible, por lo que en realidad actúa como un garante de la interoperabilidad de otras ontologías, más que como una ontología que se pueda utilizar para un propósito práctico. Entre las clases abstractas más importantes destacan las de agente, artefacto, acción, temporalidad y estado, con sus respectivas propiedades.

El proyecto aceMedia (Kompatsiaris et al, 2004) utiliza una ontología para definir la estructura y otra para representar la descripción visual, relegando los aspectos relativos a la descripción de contenidos a una tercera ontología (DOLCE) de acuerdo con principios similares a los establecidos en Harmony. DOLCE proporciona por tanto un conjunto de clases abstractas que se desarrollarán en ontologías de dominio, que son las que especificarán clases y propiedades para representar objetos, agentes y relaciones de aplicación para dicho dominio. El proyecto SmartWeb (Reithinger et al, 2005) utiliza un enfoque similar para recuperación de información multimedia en Internet, aunque basándose en una ampliación de DOLCE mediante SUMO (Niles y Peace, 2001), una meta-ontología de carácter genérico.

El proyecto BOEMIE (Kosmopoulos et al, 2006) está mucho menos restringido por el esquema de MPEG-7, por lo que tiene un grado de formalización bastante mayor que el 
resto de los proyectos revisados en esta sección. El enlace entre la descripción de los aspectos de bajo nivel (desarrollados en la ontología MDO), la estructura (desarrollada en la ontología MCO) y las clases específicas del dominio a representar se lleva a cabo a través de tan sólo dos propiedades (una en cada ontología), por lo que la descripción de los contenidos depende por completo de las ontologías de dominio (no como en el ejemplo anterior).

Los autores del proyecto ofrecen no obstante dos ontologías de dominio para ejemplificar el uso del modelo, AEO, sobre conceptos relativos a la redifusión de competiciones de atletismo y GIO, para la descripción de entidades geográficas y espaciales. Ambas están disponibles en: http://www.boemie.org/ontologies, y constituyen la aportación más valiosa a nuestro proyecto, dado que las ontologías sobre la estructura y aspectos de bajo nivel están en lo fundamental fuera del enfoque del proyecto.

El proyecto DS-MIRF (Tsinaraki, Polydoros, y Christodoulakis, 2007) proporciona una arquitectura que se basa en una meta-ontología (Upper Ontology) que permite el establecimiento de correspondencias directas con el conjunto de MPEG-7. Sobre la base de esta meta-ontología, se pueden crear ontologías de aplicación que permiten interrelacionar servicios o procesos no presentes en la especificación de MPEG-7 con descripciones multimedia que sigan el estándar. Así mismo, el proyecto ha desarrollado una metodología específica para el desarrollo de ontologías de dominio (en combinación o no con ontologías de aplicación) que es de especial interés para nuestro proyecto (Tsinaraki, Polydoros, y Christodoulakis, 2004).

El trabajo del grupo Rhizomik se basa en el desarrollado a lo largo del proyecto DS-MIRF (García et al, 2008a) y comparte una estrategia común, en el sentido de que ambas intentan proporcionar una correspondencia 1 a 1 con respecto del estándar ISO. Sin embargo, la parte más interesante del trabajo de este grupo (desde nuestra perspectiva) está relacionada con la representación de descripciones para prensa.

Arndt, Troncy, Staab, Hardman y Vacura (2007) describen la ontología COMM que 
normaliza el proceso de asignación de descripciones a contenido para MPEG-7. Las ideas básicas sobre cómo afrontar el problema de representar MPEG-7 en el ámbito de la Web Semántica son similares (de hecho se basa en DOLCE), aunque la integración de las ontologías de dominio que deben proporcionar las clases e instancias con las que llevar a cabo las descripciones también se formalizan de manera explícita, lo que permite por ejemplo distinguir entre descripciones llevadas a cabo manual y automáticamente.

Iniciativas relacionas con la aplicación de tecnologías de la Web Semántica a la documentación audiovisual en el archivo de televisión

\section{a) Proyecto MuNCH}

El proyecto MuNCH es una iniciativa del "Netherlands Institute for Sound and Vision" (NISV) y varias universidades en el que se utilizan tecnologías de la Web Semántica como las ontologías o el razonamiento automatizado en conjunción con la transcripción automatizada del habla, la búsqueda conceptual sobre material audiovisual y los productos documentales típicos como las descripciones de planos y la indización mediante un tesauro especializado.

Hollink y otros (2009) dan cuenta de las principales características del proyecto, que nosotros resumiremos aquí. El enfoque fundamental de este proyecto se deriva de la concepción patrimonial del material audiovisual y de las funciones de conservación y difusión de dicho patrimonio por parte del NISV. Esta es una perspectiva de interés para el presente proyecto, que además se ve enriquecida por el hecho de que el NISV también da servicio a profesionales, como productores y periodistas de diversos medios Holandeses. El acceso de estos usuarios al archivo tiene básicamente los mismos objetivos (conseguir material ilustrativo e informativo) que el que tiene lugar dentro de un archivo televisivo al uso (Hollink et al. 2009), por lo que el proyecto en cuestión es doblemente interesante desde nuestra perspectiva.

El NISV es una institución nacional que actúa como archivo audiovisual para todas las cadenas de televisión holandesas. Actualmente se hallan inmersos en un proceso de 
digitalización retrospectiva cuyo objetivo último es hacer públicos y accesibles los contenidos de más de 700.000 horas de vídeo. El ritmo de digitalización retrospectiva es de unas 20.000 horas al año, mientras que cada año se integran al archivo unas 8.000 horas de nuevos contenidos.

Antes del comienzo del proyecto $\mathrm{MuNCH}$ el tratamiento documental se realizaba de forma tradicional, es decir, mediante la descripción basada en planos y la indización y resumen de los documentos en su conjunto (Hollink et. al, 2009). Debido a la ingente cantidad de horas de vídeo a procesar, las descripciones de planos son bastante escasas y priman la indización (que se realiza utilizando el tesauro GTAA) y el resumen.

Esto ocasiona problemas relacionados con la recuperación de secuencias (Huurnink et. al, 2010), y es uno de los aspectos que justifica la posibilidad de semi-automatizar el tratamiento de los materiales audiovisuales. En este sentido, el proyecto MuNCH ha trabajado sobre la utilización de tecnologías para la indización automática basándose en el reconocimiento automático del habla, así como en la búsqueda conceptual sobre vídeo. Estos aspectos deberían permitir aumentar la utilidad de las descripciones sobre las que se basa la recuperación, y facilitar el acceso a nivel de secuencia.

Desde el punto de vista de las tecnologías de la Web Semántica, la principal aportación de este proyecto está relacionada con la utilización de técnicas de razonamiento automático sencillas para la asociación de las consultas de los usuarios con los "detectores de conceptos" del sistema de recuperación conceptual de vídeo a través de un tesauro (Hollink et al, 2009. Básicamente se trata de explotar la estructura jerárquica y asociativa de un tesauro para enlazar el nivel léxico de la consulta con el patrón del sistema que mejor la representa, lo cual constituye una buena alternativa a la búsqueda basada en seleccionar "imágenes parecidas".

Por otra parte, se utilizó la ontología WordNet (Fellbaum, 1998) para enriquecer el tesauro GTAA que se empleaba tradicionalmente en el archivo (Hollink et. al, 2008). La utilización de la estructura generada automáticamente es comparable en eficacia a la utilización de la estructura original (Hollink et. al, 2009), aunque la utilización conjunta 
de ambas estructuras ha resultado en mejoras modestas que no permiten concluir si esta es una forma realmente útil de expandir un tesauro. Parece ser que la utilización de WordNet es fundamentalmente útil para añadir estructura a lenguajes controlados no jerárquicos, más que para expandir la estructura de los ya existentes.

\section{b) Proyecto S5T}

El proyecto S5T (Castells et. al, 2007) persigue el establecimiento de modelos y algoritmos para la búsqueda semántica, basados en la modelización del conocimiento de un dominio mediante ontologías, la anotación semi-automática basada en dichas ontologías y la aplicación de algoritmos de indización y ranking basados en principios semánticos.

Una parte del trabajo se ha centrado en la aplicación de tecnologías relacionadas con el reconocimiento automático del habla para la extracción de textos de material de audio. En concreto se utiliza la técnica de "word spotting" o reconocimiento del habla basado en palabras clave (Tejedor et. al, 2006). Esta técnica es ventajosa dado que se centra tan sólo en un diccionario limitado, que puede estar compuesto perfectamente por los términos del tesauro, o en su caso de los términos de una ontología (Tejedor et. al, 2007). Sin embargo, esta ventaja en la eficacia de la recuperación puede ser contrarrestada (o incluso sobrepasada) por la desventaja que acarrea la necesidad de repetir todo el proceso cuando el tesauro cambie, se actualice o simplemente crezca, lo que ocurrirá sin duda múltiples veces a lo largo de la vida de éste.

La utilización de una ontología en lugar de un tesauro evitará varios de los problemas asociados a este método, como los cambios en la estructura y las relaciones entre los conceptos, que hipotéticamente se pueden hacer trazables retrospectivamente. En otras palabras, se puede mantener una relación explícita de los cambios acaecidos en la estructura, semántica y relaciones de la ontología a lo largo del tiempo, lo que no obstante puede ser bastante intensivo en términos de trabajo.

Sin embargo, a medida que se amplíe el léxico de la ontología/tesauro, se abrirá una 
distancia cada vez mayor entre las descripciones asociadas a los documentos audiovisuales y el estado actual del lenguaje controlado. De esta forma, el acercamiento tradicional basado en grandes diccionarios podría proporcionar una mejor base de partida. En cualquier caso, se trata de una aportación interesante que merece la pena considerar, sobre todo cuando los problemas fundamentales de recuperación se refieran a la falta de precisión del sistema.

\section{c) Semantic Television}

"Semantic television is defined as accessing and processing existing knowledge as well as creating new TV related content and information pools formalized using semantic web techniques and methods." (Nern et. al, 2008a). Joachim Nern definió formalmente el concepto a partir de las ideas de Dowman (2005). El propio autor ha presentado varios trabajos sobre la materia en EUROMEDIA, una conferencia sobre modelado y simulación tecnológica. Estas contribuciones se centran en la utilización de "servicios Web" y arquitecturas orientadas a servicio (SOA) para la gestión del flujo de trabajo en televisión (Atanasova et al, 2007). En estas contribuciones se describe con bastante poco detalle el uso que se hace de ontologías y demás tecnologías de la Web Semántica para el tratamiento documental, mientras que pueden ser de más utilidad desde una perspectiva de ingeniería del software, que no obstante está fuera del enfoque de este trabajo. Sin embargo, sí demuestran una continuidad del concepto de Televisión Semántica utilizado por Dowman (2005) y que recientemente ha dado lugar a la iniciativa NoTube.

NoTube es un proyecto financiado por la Comisión Europea (programa eContentPlus) que se inserta en una corriente más general basada en la integración de la Web y la Televisión digital (Notube 2010). En este sentido, es un proyecto bastante amplio que trabaja sobre la base de tecnologías de la Web Semántica para la integración de contenidos televisivos como "Linked Open Data" (Schopman et. al, 2010). Es interesante por tanto desde el punto de vista de la difusión, que ocupa un lugar secundario en este proyecto, aunque no se centra en aspectos relativos al tratamiento documental de materiales audiovisuales. 
d) VideoActive

Según Fernández-Quijada y Frontino (2009) VideoActive es "...un proyecto colaborativo paneuropeo con una aproximación temática tanto a la historia de Europa a través de la televisión como a la historia de la televisión europea, que tiene entre sus metas establecer puentes de colaboración con el mundo académico".

El proyecto VideoActive está fundamentalmente centrado en la difusión del patrimonio audiovisual europeo, por lo que su relación con este proyecto es colateral, aunque es de interés para nuestro propósito la utilización de tecnologías derivadas de la Web Semántica como parte fundamental del mismo (Oomen et al, 2009).

En este sentido, Oomen (Oomen et. al, 2010) describe la aplicación de tecnologías de la Web Semántica en el proyecto en dos ejes, la recuperación de metadatos en RDF a través de SPARQL y la integración de metadatos mediante la utilización de un Schema RDF propio (basado en Dublin Core) y el tesauro multilingüe ThesauriX según la especificación SKOS. Es interesante hacer notar que los autores afirman que las capacidades de razonamiento sobre la estructura RDF que proporciona esta arquitectura facilitan notablemente la importación e integración de metadatos provenientes de los diferentes proveedores de contenidos, fundamentalmente cadenas públicas europeas y permiten explotar las relaciones de un tesauro expresado en SKOS para mejorar los resultados de las búsquedas (Oomen et. Al, 2010).

\section{Conclusiones}

Se ha llevado a cabo a lo largo del trabajo una visión general de la relación entre la Web Semántica y los archivos de televisión. Cabe destacar que aunque existen muchos trabajos relacionados con la información audiovisual, especialmente la sonora musical, es mucho menor el número de trabajos y desarrollos realizados en materia de los archivos de televisión. 
Son muchas y variadas las líneas de trabajos que pueden llevarse a cabo sobre la Web Semántica con el fin de agilizar y facilitar la información audiovisual. Hemos de recordar que la descripción en los archivos de televisión sigue siendo manual al igual que la indización de dichos contenidos, y que la indización cuenta con graves problemas en los lenguajes de indización ya que es difícil adaptar herramientas documentales tradicionales a la descripción de acciones, que no conceptos, que deben recoger las herramientas de descripción televisiva. Y es en este punto en el que la Web Semántica aporta una visión integradora e innovadora para la identificación de contenidos.

\section{Reconocimiento}

Este trabajo ha sido financiado por el Gobierno de Extremadura (Consejería de Educación, Ciencia y Tecnología) y el Fondo Social Europeo dentro del plan de apoyo a las actuaciones de los Grupos de Investigación inscritos en el catálogo de la Junta de Extremadura. GR10019.

\section{Referencias bibliográficas}

ALAM, S., IQBAL, Z., NOLL, J. \& CHOWDHURY, M. M. R. (2009) Semantic Personalization Framework for Connected Set-Top Box Environment. En: O Dini, M Hoffmann, V Klyuev, J Noll \& W Su. (eds.), Second International Conference on advantages in human-oriented and personalized mechanism, technologies, and services. Nueva York: IEEE, pp. 97-102.

ARNDT, R., TRONCY, R., STAAB, S., HARDMAN, L. \& VACURA, M. (2007) COMM: Designing a well-founded multimedia ontology for the web. En: K Aberer, KS Choi, N Noy, D Allemang, KI Lee, L Nixon, J Golbeck, P Mika, D Maynard, R Mizoguchi, G Schreiber \& P CudreMauroux, (eds)., 'Semantic Web, Proceedings' Berlín: Springer-Verlag, pp. 30-43.

ATANASOVA, T., NERN, H. J., DZIECH, A. \& SGOUROS, N. M. (2007) Framework approach for search and meta-data handling of AV objects in digital TV cycles. En: $L$ Rothkrantz \& $C$ VanderMast, (eds.), EUROMEDIA 2007. Gante: Universidad de Gante, pp. 145-147. 
AVRITHIS, Y., O'CONNOR, N. E., STAAB, S. \& TRONCY, R. (2008) 'Introduction to the special issue on "Semantic Multimedia"'. Journal of Web Semantics, 6(2), pp. 137-138.

BELLEKENS, P., HOUBEN, GJ., AROYO, L., SCHAAP, K. \& KAPTEIN, A. (2009) User Model Elicitation and Enrichment for Context-sensitive Personalization in a Multiplatform TV Environment. En: 'Euroitv'09: proceedings of the seventh European Interactive Television Conference'. Nueva York: ASSOC COMPUTING MACHINERY, pp. 119-128.

BERNERS-LEE, T. (2004) Transcript of Tim Berners-Lee's talk to the LCS 35th Anniversary celebrations, 2004. Instituto Tecnológico de Massachussets. [en línea] http://www.w3.org/1999/04/13-tbl.html [Visitado el 5 de Abril de 2011]

BERNERS-LEE, T., HENDLER, J., LASSILA, O. (2001) 'The semantic web', Scientific american 284(5) 28--37.

BLANCO-FERNANDEZ, Y., PAZOS-ARIAS, J., GIL-SOLLA, A., RAMOS-CABRER, M., BARRAGANS-MARTINEZ, B., LOPEZ-NORES, M., GARCÍA-DUQUE, J., FERNANDEZ-VILAS, A. \& DIAZ-REDONDO, R. (2004) 'Session 9-Web Search and Personalization-AVATAR: An Advanced Multi-agent Recommender System of Personalized TV Contents by Semantic Reasoning', Lecture Notes in Computer Science 3306, 415--421.

BLANCO-FERNANDEZ, Y., PAZOS-ARIAS, J. J., GIL-SOLLA, A., RAMOS-CABRER, M., LOPEZNORES, M., GARCÍA-DUQUE, J., FERNANDEZ-VILAS, A. \& DIAZ-REDONDO, R. P. (2008) 'Exploiting synergies between semantic reasoning and personalization strategies in intelligent recommender systems: A case study', Journal of Systems and Software 81(12), 2371-2385.

BLANCO IZQUIERDO, MJ, PÓVEDA LOPEZ, IC. (2015) Metadatos documentales en Canal Extremadura Televisión. Cuadernos de Documentación Multimedia, vol. 26, pp. 113-132.

BRIASSOULI, A., DASIOPOULOU, S. \& KOMPATSIARIS, I. (2007) Ontology-based trajectory 
analysis for semantic event detection. En: ICSC 2007: International Conference on Semantic Computing, Proceedings. Los Alamitos: IEEE Computer Society, pp. 735-742.

BRUT, M., SEDES, F., GRIGORAS, R. \& CHARVILLAT, V. (2008) 'An ontology-based approach for providing multimedia personalised recommendations', International Journal of Web and grid services 4(3) 314-329.

CASTELLS, P., CANTADOR, I., COLÁS, J., DIEZ, F., FERNÁNDEZ, M., LÓPEZ-COLINO, F., MACIAS, J., O'DONNELL, M., RICO, M., RUIZ, M. \& OTHERS (2007) Scalable semantic personalized search of spoken and written contents on the Semantic Web. Informe técnico - TIN 2005-06885. Madrid: Universidad Autónoma de Madrid.

CALDERA-SERRANO, J (2006). Terminological control of "anonyous groups" for catalogues of audiovisual television documents. Journal of librarianship and information science, vol. 38, n. ${ }^{\circ} .3$, pp. 187-195

CALDERA-SERRANO, J. (2015). Externalización de la gestión documental audiovisual en las televisiones por medio de productoras audiovisuales y agencias de noticias. TransInformaçao, 27 (2), pp. 145-156

CALDERA-SERRANO, J., SÁNCHEZ-JIMENEZ, R (2008). Ontología para el control y recuperación de información onomástica en televisión, El Profesional de la Información, 17 (1), pp. 86-91

DASIOPOULOU, S., TZOUVARAS, V., KOMPATSIARIS, I. \& STRINTZIS, M. G. (2010) 'Enquiring MPEG-7 based multimedia ontologies', Multimedia tools and applications, 46(23, Sp. Iss. SI) 331-370.

DOWMAN, M., TABLAN, V., CUNNINGHAM, H., URSU, C. \& POPOV, B. (2005) 'Semantically enhanced television news through web and video integration. En: Second European Semantic Web Conference (ESWC'2005). 
FELLBAUM, C. (1998) WordNet: An electronic lexical database. Cambridge: The MIT Press.

FERNANDEZ-QUIJADA, D. \& FORTINO, M. (2009) 'Servicio público de television y patrimonio audiovisual: el proyecto VideoActive', El Profesional de la Información, 18(5), 545-551.

GARCÍA, R., MANUEL, J., PERDRIX, F., GIL, R. \& OLIVA, M. (2008a) The Rhizomer Semantic Content Management System. En: MD Lytras, JM Carroll, E Damiani \& RD Tennyson (eds.), Emerging technologies and information systems for the Knowledge Society, proceedings. Berlín: Springer-Verlag, pp. 385-394.

GROSKY, W., SREENATH, D. \& FOTOUHI, F. (2002) 'Emergent semantics and the multimedia semantic web', ACM SIGMOD Record, 31(4) 54-58.

HIDALGO GOYANES, P. (1999) Análisis documental de audiovisuales. En: García Gutiérrez, A. Introducción a la documentación informativa y periodistica. Sevilla: MAD, p.333-350.

HOLLINK, L., MALAISÉ, V. \& SCHREIBER, G. (2008) Enriching a thesaurus to improve retrieval of audiovisual documents, En: Proceedings of the 3rd International Conference on Semantic and Digital Media Technologies: Semantic Multimedia. Berlín: Springer-Verlag, pp. 47-60.

HOLLINK, L., SCHREIBER, G., HUURNINK, B., VAN LIEMPT, M., DE RIJKE, M., SMEULDERS, A., OOMEN, J. \& DE JONG, A. (2009) 'A Multidisciplinary Approach to Unlocking Television Broadcast Archives', Interdisciplinary science reviews, 34(2-3), 253-267.

HUNTER, J. (2001) Adding multimedia to the Semantic Web.: building an MPEG-7 ontology. En: Proceedings of the first Semantic Web working symposium, SWWWS'01. Palo Alto: Universidad de Stanford.

HUURNINK, B., HOLLINK, L., VAN DEN HEUVEL, W. \& DE RIJKE, M. (2010) 'Search behavior of media professionals at an audiovisual archive: A transaction log analysis', 
Journal of the American Society for Information Science and Technology, 61(6) , 1180-1197.

IFLA-UBCIM. (2001) UNIMARC manual: authorities format. Munich: UBICM Publications. Segunda Edición.

KHRIYENKO, O. (2007) 4I (for eye) multimedia - Intelligent semantically enhanced and context-ware multimedia browsing. En: S Faria (eds.), SIGMAP 2007: Proceedings of the Second International Conference on Signal Processing and Multimedia Applications. Setubal: INSTICC, pp. 233-240.

KOMPATSIARIS, I., AVRITHIS, Y., HOBSON, P., MAY, T. \& TROMP, J. (2004) 'Achieving Integration of Knowledge and Content Technologies: The AceMedia Project' En: Universidad Nacional Técnica de Atenas (ed), Proceedings of the European Workshop on the Integration of Knowledge, Semantics and Digital Media Technology. Londres: Royal Statistical Society.

KOSMOPOULOS, D., KARKALETSIS, V., PERANTONIS, S., PALIOURAS, G. \& SPYROPOULOS, C. (2006) 'Representation and Analysis of Multimedia Content: The BOEMIE Proposal' En: Proceedings of the Workshop: Crossing media for improved information access. Génova: Consorcio Reveal This, pp 24-26.

LAGOZE, C. \& HUNTER, J. (2001) 'The ABC ontology and model'. Journal of Digital Information, 2(2), ---.

MANOLA, F. \& MILLER, E. (2004) RDF Primer. Recomendación W3C del 10 de Febrero de 2004. [en línea] http://www.w3.org/TR/rdf-primer/ [Visitado el 5 de Abril de 2011]

MARTÍNEZ, J. M. (Ed.) (2004) MPEG-7 Overview (version 10). ISO/IEC JTC1/SC29/WG11 (MPEG) documento no. N6828, Octubre 2004.

MOUTSELAKIS, E. V. \& KARAKOS, A. S. (2009) Semantic Web multimedia Metadata 
retrieval: A music approach. En: V Chrissikopoulos, N Alexandris, C Douligeris \& S Sioutas (eds.), 13th Panhellenic Conference on Informatics, Proceedings. Los Alamitos: IEEE Computer Society, pp. 43-47.

NERN, H. J., ATANASOVA, T. \& JESDINSKY, G. (2008a) Semantic television - A new vision or a new business case approach? Interactive media based edutainment realized as web 3.0 environment. En: JMRS Tavares \& RN Jorge (eds.), EUROMEDIA 2008. Gante: Universidad de Gante, pp. 153-156.

NILES, I. \& PEASE, A. (2001) 'Towards a standard upper ontology'. Proceedings of the international conference on Formal Ontology in Information Systems, 2001, 2-9.

NOTUBE NETWORK (2010) Notube annual report 2010. Informe técnico. 2010. [en línea] http://cordis.europa.eu/fp7/ict/content-knowledge/docs/notube-annual-report2010.pdf [Visitado el 1 de Mayo de 2011]

OOMEN, J., TZOUVARAS, V., HECHT, A., CONESA, A. \& FORTINO, M. (2010) 'Video Active: European television heritage online', Catalan Journal of Communication \& Cultural Studies, 2(1), 93--100.

POLO, A. (2005) M-ADVANTAGE - Multimedia - Automatic digital video \& audio network through advanced publishing European service. En: M Bramer \& V Terziyan (eds.), Industrial Applications of Semantic Web. Nueva York: Springer, pp. 199-212.

POLO-CARRIÓN, JA, CALDERA-SERRANO, J, PÓVEDA-LÓPEZ, IC (2011). Metadatos y audiovisual: iniciativas, esquemas y estándares. Documentación de las Ciencias de la Información, vol. 34, pp. 45-64.

REITHINGER, N., BERGWEILER, S., ENGEL, R., HERZOG, G., PFLEGER, N., ROMANELLI, M. \& SONNTAG, D. (2005) A look under the hood: design and development of the first SmartWeb system demonstrator. En: Proceedings of the 7 th international conference on Multimodal interfaces. Nueva York: ACM, pp. 159-166. 
TEJEDOR, J., BOLACOS, D., GARRIDO, J. \& COLÁS, J. (2006) 'Búsqueda y extracción de información en Audio Mining'. En: IADIS International Conference on WWW/Internet. Murcia: ladis.

TEJEDOR, J., GARCÍA, R., FERNANDEZ, M., LÓPEZ-COLINO, F., PERDRIX, F., MACIAS, J., GIL, R., OLIVA, M., MOYA, D., COLÁS, J. \& OTHERS (2001) 'Ontology-based retrieval of human speech. En: Database and Expert Systems Applications, 2007. Regensburg: IEEE, 485-489.

TSINARAKI, C., POLYDOROS, P. \& CHRISTODOULAKIS, S. (2004) Integration of OWL ontologies in MPEG-7 and TV-Anytime compliant Semantic Indexing. En: Proceedings of the 16th International Conference on Advanced Information Systems Engineering. Riga: Riga Technial University, pp. 398-413.

TUMMARELlO, G., MORBIDONI, C., PULITI, P. \& PIAZZA, F. (2005) Semantic audio hyperlinking: a multimedia-Semantic Web scenario. En: P Nesi, K Ng \& J Delgado (eds.), First International Conference on Automated Production of Cross Media Content for Multichannel Distribution, Proceedings. Los Alamitos: IEEE Computer Society, pp. 111-114.

W3C (2011) Resource Description Framework (RDF). [en línea] http://www.w3.org/RDF/. [Visitado el 1 de Mayo de 2011] 